\title{
Mild External Compression of the Leg Increases Skin and Muscle Microvascular Blood Flow and Muscle Oxygenation during Simulated Venous Hypertension
}

\author{
T. B. Neuschwander, ${ }^{1}$ B. R. Macias, ${ }^{1}$ A. R. Hargens, ${ }^{1}$ and Q. Zhang $^{2}$ \\ ${ }^{1}$ Department of Orthopaedic Surgery, University of California-San Diego, San Diego, CA 92093, USA \\ ${ }^{2}$ Department of Orthopaedics, Sahlgrenska University Hospital, Per Dubbsgatan 14, 6tr, 41346 Göteborg, Sweden
}

Correspondence should be addressed to Q. Zhang, qiuxia.zhang@orthop.gu.se

Received 6 November 2012; Accepted 22 November 2012

Academic Editors: A. Hirata, A. Suzuki, and Y. Tohno

Copyright (C 2012 T. B. Neuschwander et al. This is an open access article distributed under the Creative Commons Attribution License, which permits unrestricted use, distribution, and reproduction in any medium, provided the original work is properly cited.

\begin{abstract}
We studied the effect of mild external leg compression on both skin and muscle microvascular flow, and muscle oxygenation in the leg of healthy subjects during simulated venous hypertension. Skin and muscle microvascular blood flows were measured using photoplethysmography (PPG), and muscle oxygenation was measured using near-infrared spectroscopy (NIRS). Both PPG and NIRS probes were placed over the anterior compartment of the right leg in 8 healthy subjects. Measurements were taken under three experimental conditions: external leg compression $(40 \mathrm{mmHg})$; simulated venous hypertension $(65 \mathrm{mmHg}$ thigh cuff); external leg compression during simulated venous hypertension. Muscle oxygenation was measured only under external leg compression during simulated venous hypertension. Simulated venous hypertension decreased skin and muscle microvascular blood flows from $100 \%$ (baseline) to $35.8 \pm 2.9 \%$ and $31.9 \pm 1.3 \%(P<0.001)$, respectively. External leg compression during simulated venous hypertension caused 2 -fold increases in both skin and muscle microvascular blood flows compared to simulated venous hypertension $(P<0.001)$. Similarly, external leg compression during simulated venous hypertension significantly restored muscle oxygenation by $23 \pm 7 \%$ compared to its baseline $(P<0.05)$. Our results demonstrate that mild external leg compression counteracts the decreases in skin microvascular flow, muscle microvascular flow, and muscle oxygenation induced by simulated venous hypertension in the leg.
\end{abstract}

\section{Introduction}

Venous hypertension is associated with decreased capillary density, arterial inflow, and local skin blood flow of the leg [1]. Therapies utilizing external compression of the leg prevent stasis and deep venous thrombosis, decrease lower extremity edema, manage chronic venous insufficiency, increase TcPO2, and improve wound healing [2-6]. Compression garments are the most common compression therapy used in clinical practice for the treatment chronic venous insufficiency and typically operate between 15 and $60 \mathrm{mmHg}$ [7]. An optimal compression pressure around $50-60 \mathrm{mmHg}$ is reported to reduce chronic edema of the lower extremity [8]. The precise mode of action of external compression is not fully understood yet but numerous perceived benefits have been reported. Compression therapy increases both large and small vessel blood flow, in addition, compression therapy increases cutaneous capillary density $[5,9]$. Recently, it has been shown that mild external compression increases arterial inflow of the human forearm [10]. Compression also causes significant increase in leg pulsatile blood flow $[11,12]$ and microcirculatory skin blood flow [13]. However, the impact of external compression on microcirculatory muscle blood flow and muscle oxygenation remains unclear.

A newly developed photoplethysmography (PPG) technique, using green and near-infrared light sources, enables simultaneous noninvasive monitoring of skin and muscle microvascular flow. This technique is employed in the present paper and is validated in multiple studies against 
invasive single-fiber laser-Doppler flowmetry and other validation tests [14-19].

In the present study, a model of venous hypertension is used in healthy subjects to increase venous pressure, thus reducing perfusion pressure and blood perfusion of the leg. The object of the study is to determine the effect of mild external leg compression on both skin and muscle microvascular flows, and muscle oxygenation during simulated venous hypertension. We hypothesize that mild external leg compression counteracts the decreases in skin microvascular flow, muscle microvascular flow, and muscle oxygenation induced by simulated venous hypertension in the leg.

\section{Materials and Methods}

2.1. Subjects. Eight healthy subjects (4 men and 4 women, age range: 23-62) participated in this study. Subjects were active, normotensive, nonsmokers, and had no prior history of leg pain that necessitated medical intervention. The study protocol was explained to each subject. All subjects gave informed written consent prior to participation. The protocol was approved by the Human Research Institutional Review Board of the University of California, San Diego.

2.2. Microvascular Flow. Skin and muscle microvascular flows were measured simultaneously using PPG. A PPG instrument and probe (Department of Biomedical Engineering, Linköping University, Linköping, Sweden) continuously recorded changes in skin and muscle blood perfusion [1419]. PPG light from a light-emitting diode (LED) was directed toward the skin and absorbed and scattered in the tissue. A small amount of this light was received by a photodetector placed adjacent to the LED. Altered blood flow in the underlying tissue was related to variations in the photodetector signal $[20,21]$. The amplitude of the AC component of the PPG signal depends on the pulsatile pressure as well as the pulsatile blood flow, pulsatile blood volume, and the number of blood vessels in action for blood supply in a complex manner $[14,22]$. An increase in amplitude of the $\mathrm{PPG}_{\mathrm{AC}}$ signal indicates an increase in microvascular blood flow [20].

A PPG probe (ten by five centimeters) was placed on skin centrally over the middle third of the anterior compartment of the right leg with a bandage wrapped loosely around the leg to block ambient light. The bandage did not compress the leg or probe significantly. The probe consisted of three photodetectors and eight light sources (light-emitting diodes, LEDs). Six LEDs emitted light of a wavelength of $560 \mathrm{~nm}$ (green light) and two LEDs (placed in a special pattern and embedded in black-colored silicone rubber) emitted light in the near-infrared region of $810 \mathrm{~nm}$. Placing the green LED $3.5 \mathrm{~mm}$ from the photodetector allowed for superficial penetration of light for monitoring skin microvascular flow. In contrast, increasing the distance to $20 \mathrm{~mm}$ between the near-infrared LED and the photodetector enabled deeper penetration of light to measure muscle microvascular flow.
The signals passed through an amplifier and were highpass filtered at $0.1 \mathrm{~Hz}$ and low-pass filtered at $28 \mathrm{~Hz}$. The output of the PPG was A/D converted (DAQCard-700, National Instruments, Stockholm, Sweden) and recorded at a sampling frequency of $60 \mathrm{~Hz}$ using a LabWindows program. A computer program was used to detect the peak-to-peak values of the $\mathrm{PPG}_{\mathrm{AC}}$ signals and provided a mean value of the peak heights of the selected recording period. The device was calibrated for each subject at rest before any measurement.

2.3. Muscle Oxygenation. Relative changes in intramuscular oxygenation were measured noninvasively by continuous dual wavelength near-infrared spectroscopy (RunMan; NIM, Philadelphia, Pennsylvania). The principle of near-infrared spectroscopy (NIRS) was previously validated and employed wavelengths different from those for PPG [23]. In brief, this device measured the reflection of light transmitted at wavelengths of 760 and $850 \mathrm{~nm}$. Deoxyhemoglobin absorbed more light at $760 \mathrm{~nm}$, whereas oxyhemoglobin absorbed more light at $850 \mathrm{~nm}$. The difference in magnitude of light reflected at these two wavelengths indicated a change in the concentration of oxygenated hemoglobin and myoglobin. The device was calibrated for each subject at rest before the measurements. The RunMan near-infrared probe (ten by four centimeters) was placed on skin surface centrally over the anterior tibial muscle of the right leg, secured with tape and an elastic bandage wrapped loosely around the leg to block ambient light. The bandage did not compress the leg or probe significantly. NIRS was used to measure muscle oxygenation during simulated venous hypertension and mild external leg compression in 6 of the original 8 subjects ( 3 men, 3 women, age range: $23-62$ ). Both PPG and NIRS probes were placed on the anterior compartment approximately $5 \mathrm{~cm}$ from each other with the NIRS probe placed more distally. This distance was sufficient to ensure that PPG and NIRS measurements on the same leg did not affect each other. Muscle oxygenation was normalized to zero (\%) at minute 6 of simulated venous hypertension, as this represented a steady-state of simulated venous hypertension.

2.4. Simulated Venous Hypertension. An inflatable mid-thigh tourniquet was placed on the right thigh and inflated to $65 \mathrm{mmHg}$ to partially occlude venous return of the leg in the method of Hanna and coworkers [24].

2.5. Mild External Leg Compression. An inflatable cuff was placed over the right leg, covering the both PPG and NIRS probes. This cuff extended from the tibial tuberosity to the distal $1 / 3$ of the compartment and was inflated to $40 \mathrm{mmHg}$ [8], allowing compression of the underlying skin and anterior compartment during supine posture.

2.6. Experimental Protocol. Each subject underwent three experimental conditions, the order of which was randomized (Figure 1). All conditions were performed on the right leg for $17 \mathrm{~min}$ with $15 \mathrm{~min}$ of rest between each condition to ensure the blood flow returned to baseline and to eliminate any confounding effects of order. 


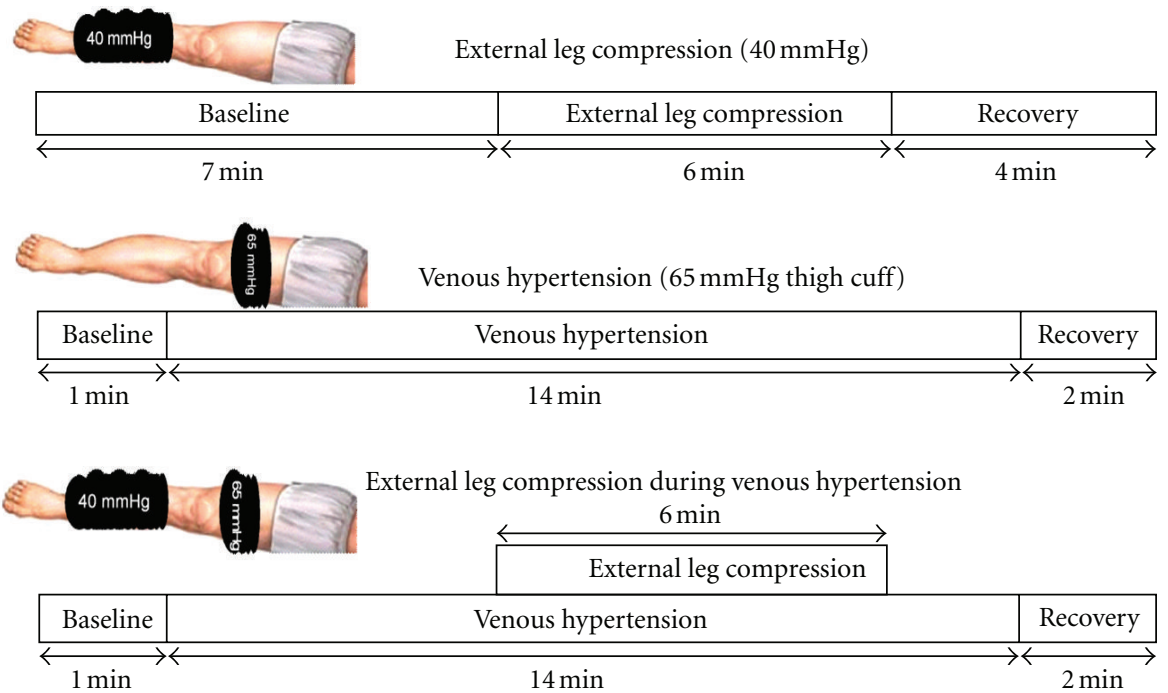

Figure 1: Schematic of the experimental conditions: external leg compression of $40 \mathrm{mmHg}$; venous hypertension (65 mmHg thigh cuff); external leg compression during venous hypertension. Anterior tibial skin and muscle microvascular blood flows were measured continuously using photoplethysmography (PPG), and muscle oxygenation was measured using near-infrared spectroscopy (NIRS) in the right leg of healthy subjects. Both PPG and NIRS probes were placed over the anterior compartment approximately $5 \mathrm{~cm}$ from each other.

External leg compression: after $7 \mathrm{~min}$ of baseline, the leg cuff was inflated to $40 \mathrm{mmHg}$ for $6 \mathrm{~min}$, followed by $4 \mathrm{~min}$ of recovery.

Simulated venous hypertension: after taking baseline, the thigh tourniquet was inflated to $65 \mathrm{mmHg}$ for $14 \mathrm{~min}$, followed by $2 \mathrm{~min}$ of recovery.

External leg compression during venous hypertension: following baseline measurements, the thigh tourniquet was inflated to $65 \mathrm{mmHg}$ to simulate venous hypertension. Simulated venous hypertension measurements were collected continuously. To counteract changes induced by $6 \mathrm{~min}$ simulated venous hypertension, the external leg compression cuff was inflated to $40 \mathrm{mmHg}$ and remained inflated for 6 $\min$. To test if there were any residual beneficial effects of external compression the leg compression cuff was deflated. Lastly, the thigh tourniquet was deflated for $2 \mathrm{~min}$ recovery measures.

2.7. Data Analysis. Microvascular flow and muscle oxygenation values are reported as means \pm SE. For each subject, the mean PPG value obtained at the initial resting period was used as a reference value. All subsequent PPG data were normalized by dividing the reference value and multiplying by 100 , thus giving an initial value of $100 \%$ for normalized data. All data were subjected to a ShapiroWilk test of normality, and a repeated-measures ANOVA was used for statistical comparisons, with the level of statistical significance set at $P<0.05$.

\section{Results}

3.1. Skin Microvascular Blood Flow. External leg compression of $40 \mathrm{mmHg}$ alone in a normal healthy leg did not significantly affect skin perfusion. Simulated venous hypertension decreased skin microvascular flow from $100 \%$ to $35.8 \pm 2.9 \%$ (Figure 2(a); $P<0.001)$. External leg compression during simulated venous hypertension increased skin microvascular flow by 2 -fold compared to the simulated venous hypertension alone $(P<0.001)$. Thus, mild external leg compression during simulated venous hypertension restored skin microvascular blood flow by about $50 \%$.

3.2. Muscle Microvascular Blood Flow. As demonstrated in Figure 2(b), $40 \mathrm{mmHg}$ external leg compression alone did not significantly affect muscle perfusion; although not significant, the mean values were higher with external pressure compared to the mean value immediately after application and at the release of mild external leg compression. Simulated venous hypertension decreased muscle microvascular flow from $100 \%$ to $31.9 \pm 1.3 \%(P<0.001)$. Simulated venous hypertension with subsequent mild external leg compression doubled muscle microvascular flow compared to the simulated venous hypertension alone $(P<0.001)$. Thus, mild external leg compression during simulated venous hypertension restored microvascular flow by about $50 \%$.

3.3. Muscle Oxygenation. Mild external leg compression partially counteracted decrements in muscle oxygenation during simulated venous hypertension. Muscle oxygenation increased by $23 \pm 7 \%$ when mild external leg compression was applied to the leg during venous hypertension (Figure 3; $P<0.05$, comparing $6 \mathrm{~min}$ data point to data points at 7-12 min). Moreover, mild external leg compression during simulated venous hypertension restored muscle oxygenation values to levels observed without venous hypertension (Figure 3: minutes 15-16). 


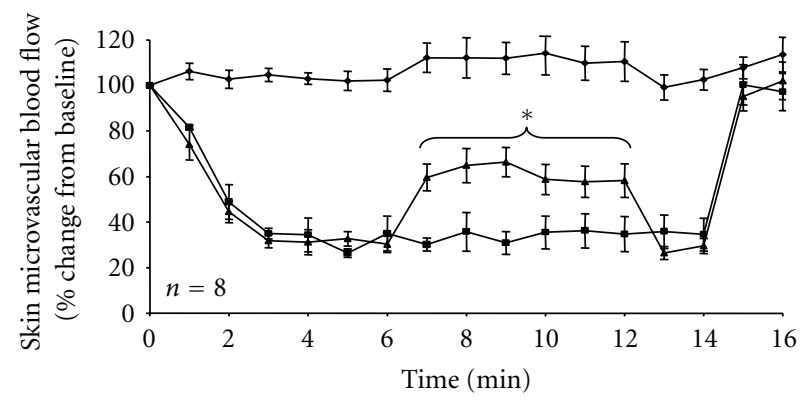

(a)

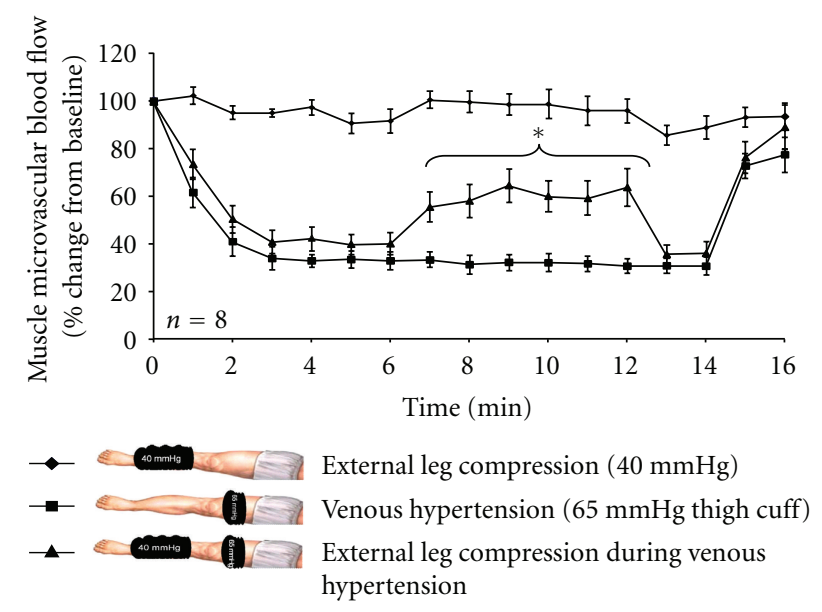

(b)

Figure 2: Anterior tibial skin (a) and muscle (b) microvascular blood flows measured by photoplethysmography (PPG) during 3 experimental conditions. Mild external leg compression of $40 \mathrm{mmHg}$ alone does not affect skin and muscle perfusion in a supine healthy subject. Simulated venous hypertension using a $65 \mathrm{mmHg}$ thigh tourniquet decreases skin microvascular blood flow by $62 \%$ and muscle microvascular blood flow by $68 \%$. Mild external leg compression of $40 \mathrm{mmHg}$ during venous hypertension doubles skin microvascular blood flow compared to the simulated venous hypertension alone. ${ }^{*} P<0.001$ repeated measures ANOVA.

\section{Discussion}

The present study demonstrates that mild external leg compression therapy during simulated venous hypertension significantly increases skin and muscle microvascular blood flows, and muscle oxygenation as compared to mild external leg compression or simulated venous hypertension alone.

The venous hypertension model, $65 \mathrm{mmHg}$ thigh cuff, employed in the present study reduces both skin and muscle microvascular flows. These blood flow results can be explained by the local reflex mechanism of the skin and muscle microvasculature $[25,26]$. A similar venous hypertension model utilized by Hanna and coworkers [24] also document about $60 \%$ decrease in skin oxygenation and $25 \%$ decrease in skin microvascular blood flow of the healthy human leg. However, they do not report the affects of venous hypertension on muscle blood flow or muscle oxygenation. In addition, they report that the ankle systolic pressure does not change with proximal thigh cuff occlusion

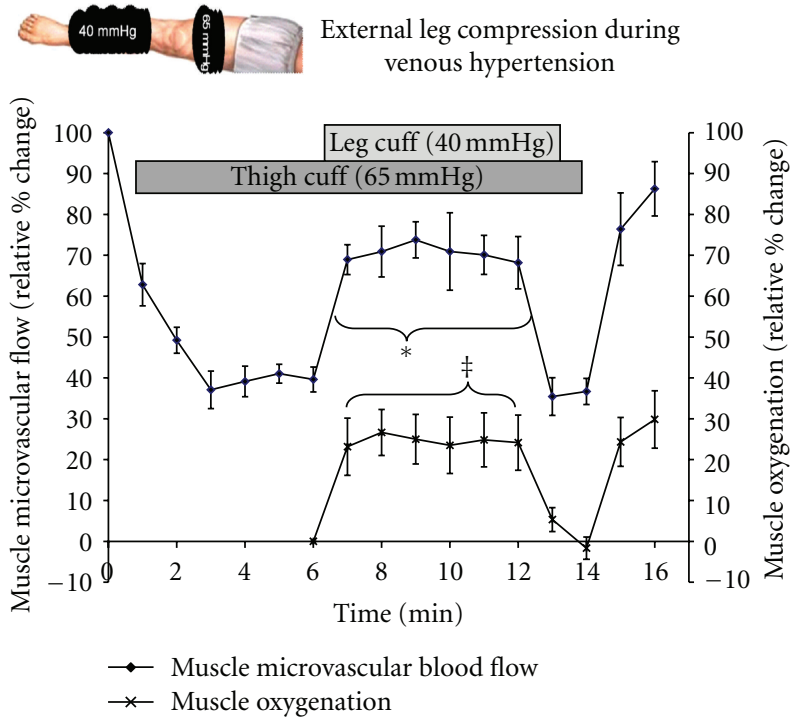

FIGURE 3: Anterior tibialis muscle microvascular blood flow measured by photoplethysmography (PPG) and muscle oxygenation measured by near-infrared spectroscopy (NIRS). External leg compression of $40 \mathrm{mmHg}$ during simulated venous hypertension using a $65 \mathrm{mmHg}$ thigh tourniquet significantly increases muscle microvascular blood flow and muscle oxygenation. In addition, muscle oxygenation levels during compression are similar to those values during recovery measures. ${ }^{*} P<0.05$ repeated measures ANOVA comparing $6 \mathrm{~min}$ data point to data points at 7-12 $\mathrm{min}$. ${ }^{\ddagger} P<0.05$ repeated measures ANOVA comparing 6 min data point to data points at $7-12 \mathrm{~min}$.

at the pressures of 40,60 , and $80 \mathrm{mmHg}$. This unchanged systolic arterial pressure coupled with the cuff pressureinduced rise in venous pressure results in a lower perfusion pressure of the leg. Therefore, venous hypertension directly impacts skin and muscle perfusion pressure, showing 64\% decrease in skin microvascular flow and $68 \%$ decrease in muscle microvascular flow.

We observed in our pilot study that external leg compression of $20-40 \mathrm{mmHg}$ during venous hypertension resulted in an increase in both skin and muscle microvascular blood flows, but external leg compression up to $60 \mathrm{mmHg}$ during venous hypertension caused a progressive decrease in both skin and muscle microvascular blood flows. Importantly, the results in the present study suggest that mild distal external leg compression $(40 \mathrm{mmHg})$ restores leg skin and muscle microvascular blood flows in a simulated venous hypertension model. Local autoregulation is a likely cause of this increase in blood flow. Reneman and coworkers [27] find increases in arteriole diameter in rabbit tenuissimus muscle when intramuscular pressure was increased by external compression. As external pressure rises from zero to the same value as perfusion pressure, they document a linear increase in arteriole diameter. An increase in arteriole diameter will increase blood flow to the tissue. Two studies by Nielsen measure muscle blood flow in healthy human leg with local ${ }^{133} \mathrm{Xe}$ washout. These studies demonstrate an increase in arteriolar dilation and a decrease in local vascular 
resistance while experiencing external compression between 0 to $20 \mathrm{mmHg}[28,29]$. Moreover, Bochmann and colleagues [10] demonstrate increases in upper extremity blood flow using custom compression garments. Our findings that mild external leg compression during venous hypertension increases both skin and muscle microvascular blood flows are in agreement with these previous studies.

Some possible mechanisms for the increases of microvascular blood flow during mild external compression with venous hypertension observed in the present study include a venoarteriolar response, venous pumping, and a myogenic effect. The venoarteriolar response is unlikely to be responsible because mild external leg compression does little to change venous pressures distal to the thigh tourniquet. The present study was conducted with subjects in the supine position to eliminate a venous pressure gradient and without voluntary muscle contraction. Therefore, mild external leg compression is unlikely to function by venous pumping or the muscle pump mechanism to increase venous return and affect skin and muscle blood flow. Moreover, the thigh tourniquet held venous pressures at $65 \mathrm{mmHg}$ so venous pumping is unlikely. Mild external compression of the leg likely decreases transmural pressure, leading to local arteriolar dilation and decreased capillary resistance. Arteriolar vessels maintain resting vascular tone in response to vessel transmural pressure. This local regulatory mechanism, known as the myogenic response, was first described by Bayliss [30]. This mechanism is an important contributor to blood flow autoregulation in response to changes in transmural pressure across the vessel wall [31]. Mayrovitz and coworkers $[11,12]$ demonstrate a significant increase in leg pulsatile blood flow during compression of the leg to an average malleolar subbandage pressure of $40.7 \pm$ $4.0 \mathrm{mmHg}$ and the mechanism for this is hypothesized to be arteriolar vasodilation due to either or both a myogenic arteriolar response or a shear-dependent venous effect. Our findings that mild external leg compression therapy during simulated venous hypertension significantly increases skin and muscle microvascular blood flows, and muscle oxygenation can be explained by local myogenic vasodilatory response to reduced arteriolar transmural pressure during external compression.

The present study was performed with subjects at rest in the supine position. Assuming that mild external leg compression during simulated venous hypertension at rest does not contract the anterior tibial muscle; changes of muscle oxygenation observed in the present study reflect muscle microvascular flow changes. Mild external leg compression during simulated venous hypertension restores both muscle microvascular flow and muscle oxygenation. Although external leg compression during simulated venous hypertension does not completely restore muscle microvascular flow, it restores muscle oxygenation. The muscle oxygenation value recorded during mild external leg compression with simulated venous hypertension is similar to the level when both thigh cuff and leg cuff were released. This result suggests that external leg compression restores muscle oxygenation to a level similar to that of intact muscle without simulated venous hypertension.
Limitations of the present study include the concern that compression of the PPG probe with the leg cuff may cause falsely elevated blood flow measurements. However, compression with a leg cuff alone caused no significant change. A second limitation of the present study is the exclusive use of noninvasive measures, namely, PPG and NIRS, both relative measurement techniques that do not provide absolute flow values, thus requiring comparison within each subject to the initial baseline values. Currently only invasive techniques are available to measure absolute microvascular flows. Therefore, independent but related noninvasive tools to measure microvascular tissue dynamics provide the best alternative to provide human tissue level responses to mechanical/pressure tissue deformations. Moreover, our skin microvascular blood flow results during partial venous occlusion demonstrate the reproducibility and reliability of the venous hypertension model in healthy subjects described by Hanna and colleagues [24]. This thigh tourniquet model of venous hypertension does not entirely simulate the inflammatory cascade, venous valve deficiency, and lack of adaptation to chronic ischemia. However, as reported previously and observed in the present study, this model effectively decreases skin and muscle microvascular blood flow in a healthy leg. Taken together, the noninvasive measurement tools employed in the present study show complementary decrements in both skin and muscle microvascular flow and muscle oxygenation during simulated venous hypertension.

\section{Conclusions}

Our data demonstrate that mild external leg compression counteracts the decrease in skin microvascular flow, muscle microvascular flow, and muscle oxygenation induced by simulated venous hypertension in the leg. Our findings explain some of the beneficial effects of external compression therapy on the treatment of venous hypertension. Future studies are needed to evaluate how the effect of mild leg compression on skin and muscle microvascular blood flows, and muscle oxygenation in the leg of patients with venous hypertension.

\section{Acknowledgments}

Funding was provided by NIH Kirschstein T32 Training Grant from the Göteborg Medical Society and the Swedish Society of Medicine.

\section{References}

[1] A. Bollinger, A. J. Leu, U. Hoffmann, and U. K. Franzeck, "Microvascular changes in venous disease: an update," Angiology, vol. 48, no. 1, pp. 27-32, 1997.

[2] J. J. Bergan and S. R. Sparks, "Non-elastic compression: an alternative in management of chronic venous insufficiency," Journal of Wound, Ostomy and Continence Nursing, vol. 27, no. 2, pp. 83-89, 2000.

[3] K. T. Delis, N. Labropoulos, A. N. Nicolaides, B. Glenville, and G. Stansby, "Effect of intermittent pneumatic foot compression on popliteal artery haemodynamics," European Journal of 
Vascular and Endovascular Surgery, vol. 19, no. 3, pp. 270-277, 2000.

[4] A. R. Eze, P. L. Cisek, B. S. Holland Jr, A. J. Comerota, R. Verramasuneni, and A. J. Comerota, "The contributions of arterial and venous volumes to increased cutaneous blood flow during leg compression," Annals of Vascular Surgery, vol. 12, no. 2, pp. 182-186, 1998.

[5] M. Jünger, A. Steins, M. Hahn, and H. M. Häfner, "Microcirculatory dysfunction in chronic venous insufficiency (CVI)," Microcirculation, vol. 7, no. 6, pp. S3-S12, 2000.

[6] R. J. Morris and J. P. Woodcock, "Evidence-based compression: prevention of stasis and deep vein thrombosis," Annals of Surgery, vol. 239, no. 2, pp. 162-171, 2004.

[7] J. T. Trent, A. Falabella, W. H. Eaglstein, and R. S. Kirsner, "Venous ulcers: pathophysiology and treatment options," Ostomy/wound management, vol. 51, no. 5, pp. 38-56, 2005.

[8] H. Partsch, R. J. Damstra, and G. Mosti, "Dose finding for an optimal compression pressure to reduce chronic edema of the extremities," International Journal of Angiology, vol. 30, no. 6, pp. 527-533, 2011.

[9] O. Agu, D. Baker, and A. M. Seifalian, "Effect of graduated compression stockings on limb oxygenation and venous function during exercise in patients with venous insufficiency," Vascular, vol. 12, no. 1, pp. 69-76, 2004.

[10] R. P. Bochmann, W. Seibel, E. Haase, V. Hietschold, H. Rödel, and A. Deussen, "External compression increases forearm perfusion," Journal of Applied Physiology, vol. 99, no. 6, pp. 2337-2344, 2005.

[11] H. N. Mayrovitz and P. B. Larsen, "Effects of compression bandaging on leg pulsatile blood flow," Clinical Physiology, vol. 17, no. 1, pp. 105-117, 1997.

[12] H. N. Mayrovitz and K. J. M. MacDonald, "Medical compression: effects on pulsatile leg blood flow," International Angiology, vol. 29, no. 5, pp. 436-441, 2010.

[13] A. Abu-Own, S. K. Shami, S. J. Chittenden, J. Farrah, J. H. Scurr, and P. D. C. Smith, "Microangiopathy of the skin and the effect of leg compression in patients with chronic venous insufficiency," Journal of Vascular Surgery, vol. 19, no. 6, pp. 1074-1083, 1994.

[14] J. Hagblad, L. G. Lindberg, A. K. Andersson et al., "A technique based on laser Doppler flowmetry and photoplethysmography for simultaneously monitoring blood flow at different tissue depths," Medical and Biological Engineering and Computing, vol. 48, no. 5, pp. 415-422, 2010.

[15] J. Mateus and A. R. Hargens, "Photoplethysmography for non-invasive in vivo measurement of bone hemodynamics," Physiological Measurement, vol. 33, no. 6, pp. 1027-1042, 2012.

[16] M. Sandberg, Q. Zhang, J. Styf, B. Gerdle, and L. G. Lindberg, "Non-invasive monitoring of muscle blood perfusion by photoplethysmography: evaluation of a new application," Acta Physiologica Scandinavica, vol. 183, no. 4, pp. 335-343, 2005.

[17] Q. Zhang, G. Andersson, L. G. Lindberg, and J. Styf, "Muscle blood flow in response to concentric muscular activity vs. passive venous compression," Acta Physiologica Scandinavica, vol. 180, no. 1, pp. 57-62, 2004.

[18] Q. Zhang, L. G. Lindberg, R. Kadefors, and J. Styf, "A noninvasive measure of changes in blood flow in the human anterior tibial muscle," European Journal of Applied Physiology, vol. 84, no. 5, pp. 448-452, 2001.

[19] Q. Zhang, J. Styf, and L. G. Lindberg, "Effects of limb elevation and increased intramuscular pressure on human tibialis anterior muscle blood flow," European Journal of Applied Physiology, vol. 85, no. 6, pp. 567-571, 2001.
[20] A. V. J. Challoner, "Photoelectric plethysmography for estimating cutanneous blood flow," in Non-Invasive Physiological Measurements, P. Rolfe, Ed., pp. 127-151, Academic Press, London, UK, 1979.

[21] A. A. R. Kamal, J. B. Harness, G. Irving, and A. J. Mearns, "Skin photoplethysmography-a review," Computer Methods and Programs in Biomedicine, vol. 28, no. 4, pp. 257-269, 1989.

[22] L. G. Lindberg and P. A. Oberg, "Optical properties of blood in motion," Optical Engineering, vol. 32, no. 2, pp. 253-258, 1993.

[23] B. Chance, S. Nioka, J. Kent et al., "Time-resolved spectroscopy of hemoglobin and myoglobin in resting and ischemic muscle," Analytical Biochemistry, vol. 174, no. 2, pp. 698-707, 1988.

[24] G. B. Hanna, D. J. Newton, D. K. Harrison, J. J. F. Belch, and P. T. McCollum, "Use of lightguide spectrophotometry to quantify skin oxygenation in a variable model of venous hypertension," British Journal of Surgery, vol. 82, no. 10, pp. 1352-1356, 1995.

[25] O. Henriksen and P. Sejrsen, "Local reflex in microcirculation in human skeletal muscle," Acta Physiologica Scandinavica, vol. 99, no. 1, pp. 19-26, 1977.

[26] O. Henriksen and P. Sejrsen, “Effect of 'vein pump' activation upon venous pressure and blood flow in human subcutaneous tissue," Acta Physiologica Scandinavica, vol. 100, no. 1, pp. 1421, 1977.

[27] R. S. Reneman, D. W. Slaaf, and L. Lindbom, "Muscle blood flow disturbances produced by simultaneously elevated venous and total muscle tissue pressure," Microvascular Research, vol. 20, no. 3, pp. 307-318, 1980.

[28] H. V. Nielsen, "Effects of externally applied compression on blood flow in subcutaneous and muscle tissue in the human supine leg," Clinical Physiology, vol. 2, no. 6, pp. 447-457, 1982.

[29] H. V. Nielsen, "External pressure-blood flow relations during limb compression in man," Acta Physiologica Scandinavica, vol. 119, no. 3, pp. 253-260, 1983.

[30] W. M. Bayliss, "On the local reactions of the arterial wall to changes of internal pressure," Journal of Physiology, vol. 28, no. 3, pp. 220-231, 1902.

[31] M. E. J. Lott, M. D. Herr, and L. I. Sinoway, "Effects of transmural pressure on brachial artery mean blood velocity dynamics in humans," Journal of Applied Physiology, vol. 93, no. 6, pp. 2137-2146, 2002. 


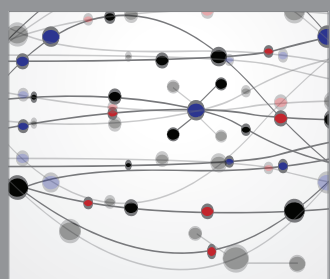

The Scientific World Journal
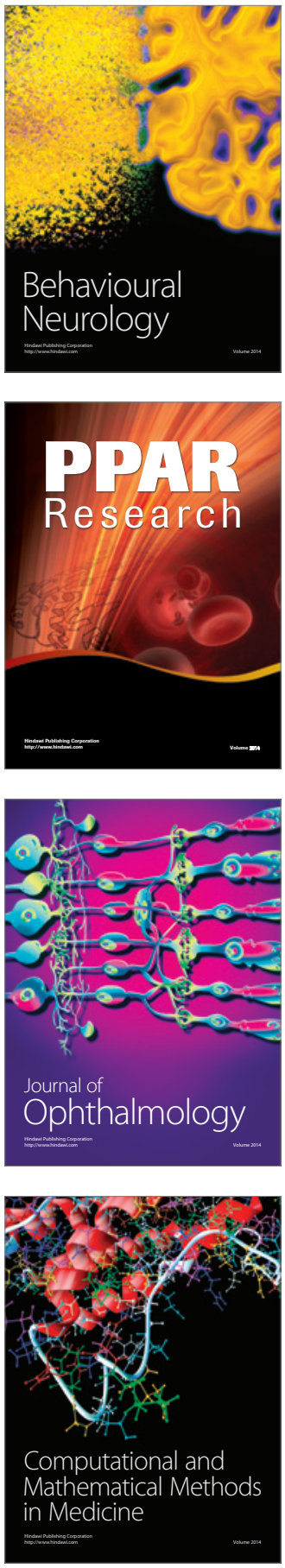

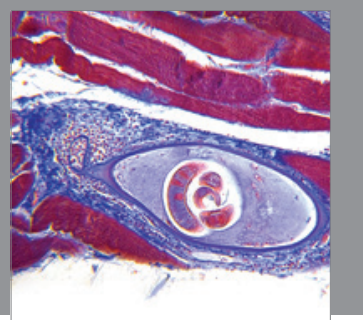

Gastroenterology

Research and Practice
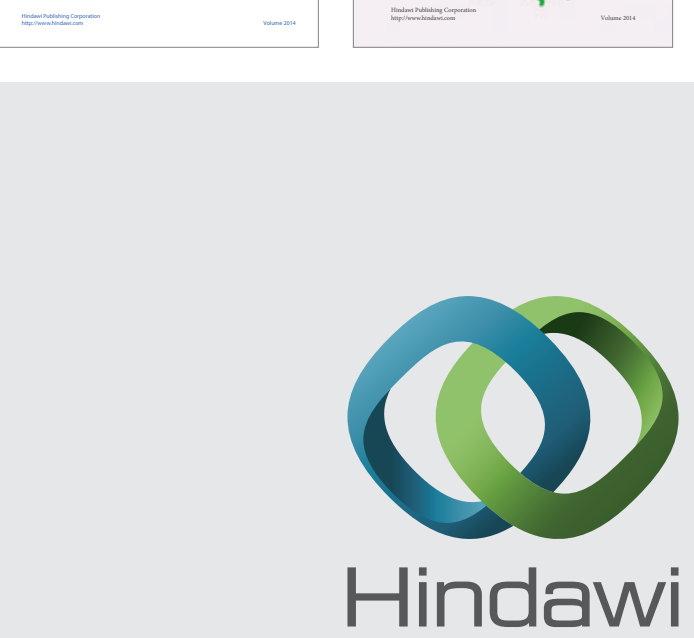

Submit your manuscripts at

http://www.hindawi.com
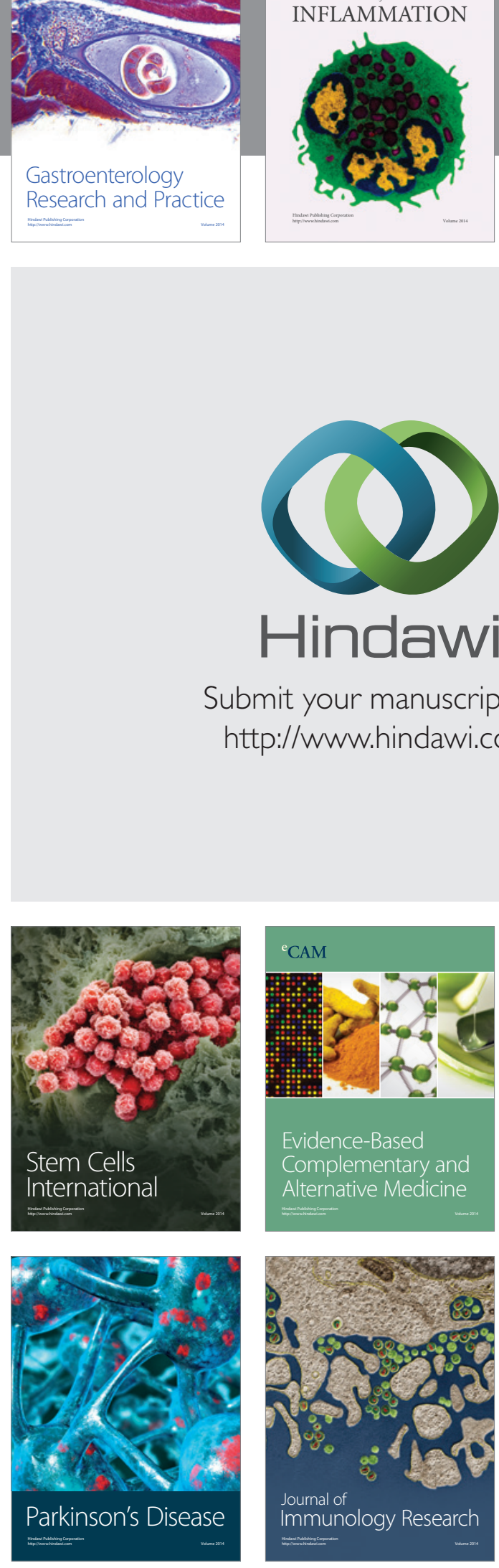

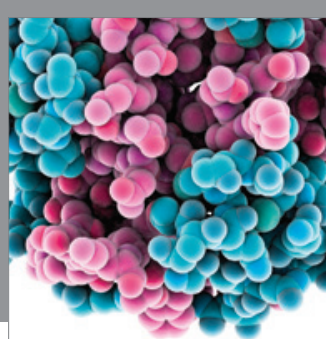

Diabetes Research
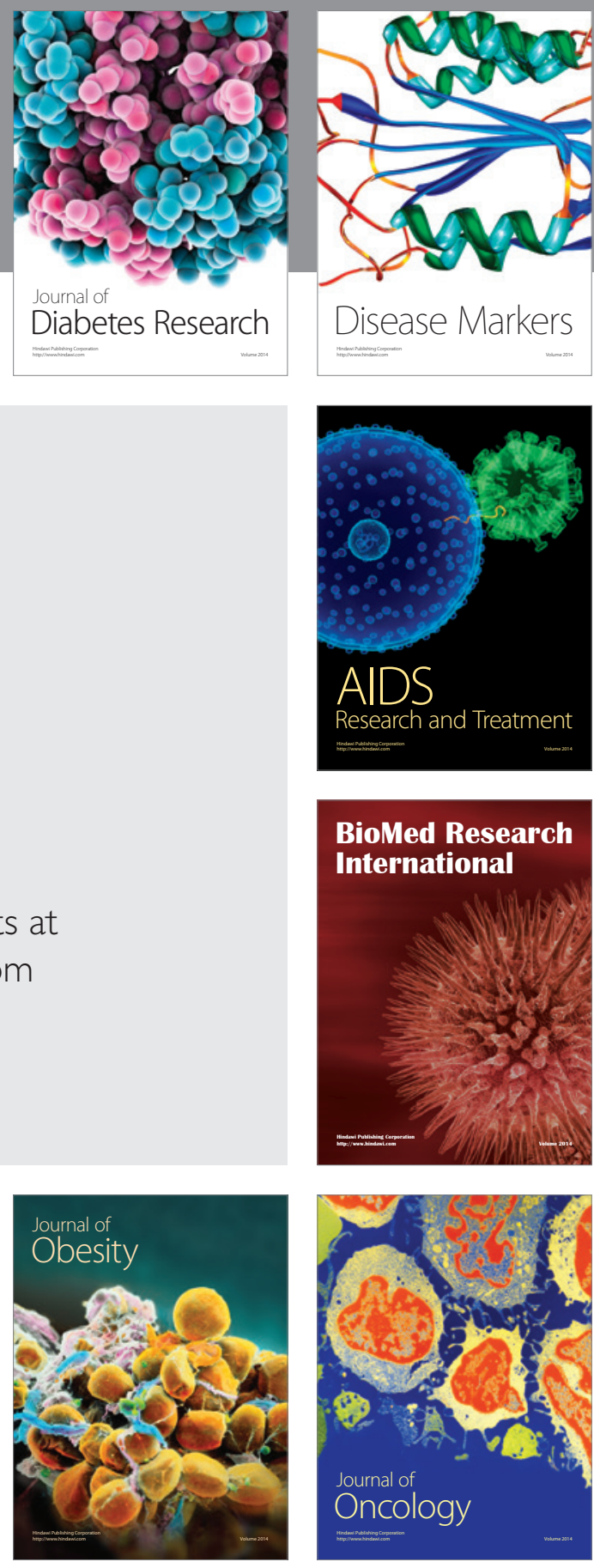

Disease Markers

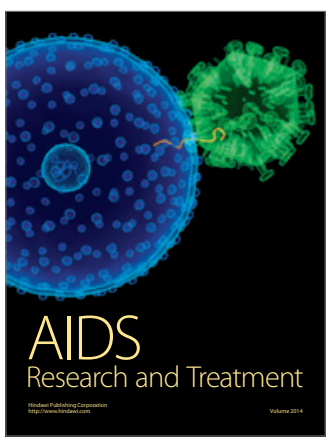

BioMed Research

International
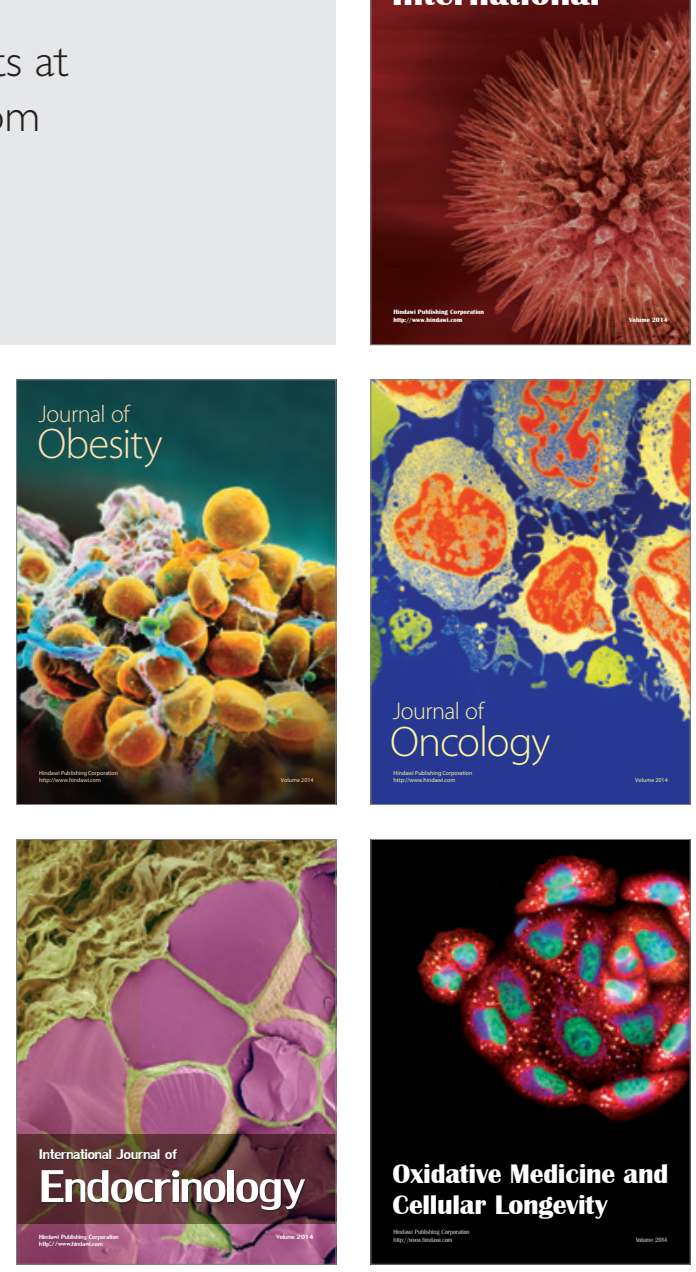